\title{
The Outcomes of Dapagliflozin Use in Real-Life Clinical Settings in Endocrinology Clinics of Islamabad, Pakistan
}

Matiullah Kamin ${ }^{1}$, Osama Ishtiaq ${ }^{2}$, Kashif Raashid ${ }^{2}$, Muhammad Umar Wahab ${ }^{3,4}$, Sajjad Ali Khan ${ }^{5}$, Umar Raja ${ }^{2}$

1. Endocrinology, Shifa International Hospital, Islamabad, PAK 2. Endocrinology, Shifa International Hospital, Islamabad, PAK 3. Endocrinology, Umar Diabetes and Footcare Clinic, Islamabad, PAK 4. Diabetology, Umar Diabetes Foundation, Islamabad, PAK 5. Endocrinology, Aga Khan University Hospital, Karachi, PAK

Corresponding author: Matiullah Kamin, matiullah_shifa@yahoo.com

\section{Abstract \\ Introduction}

Dapagliflozin is a member of a novel class of drugs (sodium-glucose cotransporter-2 inhibitors) used to treat type 2 diabetes mellitus and licensed in Pakistan in 2017. This retrospective observational study evaluated the effects of dapagliflozin on glycated hemoglobin (HbA1c) concentrations in patients treated at endocrinology clinics in Islamabad, Pakistan. The secondary objectives included assessing the effects of dapagliflozin on weight reduction and blood pressure control and to determining its safety.

\section{Methodology}

Patients with type 2 diabetes who were treated with dapagliflozin were identified by screening the electronic medical records at tertiary care hospitals in Islamabad. Data were collected at the first visit and at follow-up. Categorical variables were recorded as frequencies and percentages and compared by McNemar's tests, and continuous variables were recorded as means and standard deviations and compared by paired sample ttests.

\section{Results}

Mean $\mathrm{HbA1C}$ concentration was significantly lower at follow-up than at the first visit $(7.57 \% \pm 0.98 \%$ vs. $9.07 \% \pm 2.07 \%$, respectively; $p<0.001)$. Bodyweight $(85.09 \pm 15.92 \mathrm{~kg}$ vs. $87.07 \pm 16.11 \mathrm{~kg}$, respectively; $\mathrm{p}<0.001)$ and diastolic blood pressure $(80.34 \pm 7.12 \mathrm{mmHg}$ vs. $82.34 \pm 9.61 \mathrm{mmHg}$, respectively; $\mathrm{p}<0.001)$ were also significantly lower at follow-up than at the first visit, whereas systolic pressure showed a marginally significant reduction $(123.5 \pm 16.57 \mathrm{mmHg}$ vs. $126.83 \pm 19.97 \mathrm{mmHg}, \mathrm{p}=0.048)$.

\section{Conclusion}

Received 05/14/2020

Review began 05/27/2020 Review ended 06/04/2020 Published 06/11/2020

\section{() Copyright 2020}

Kamin et al. This is an open access article distributed under the terms of the Creative Commons Attribution License CC-BY 4.0., which permits unrestricted use, distribution, and reproduction in any medium, provided the original author and source are credited.
This first observational study of patients in Pakistan treated with dapagliflozin found that HbA1c concentration, weight, and blood pressure were reduced after initiation of dapagliflozin treatment.

Categories: Endocrinology/Diabetes/Metabolism

Keywords: type 2 diabetes mellitus, dapagliflozin, retrospective study, hemoglobin a1c

\section{Introduction}

Pakistan currently ranks fourth in the world in the percentage of people with diabetes mellitus, with an estimated prevalence of $26.3 \%$ [1]. Effective therapeutic agents are, therefore, needed to manage diabetes as well as to prevent diabetes-associated microvascular and macrovascular complications [2]. Sodium-glucose cotransporter-2 inhibitors (SGLT2i) are a novel class of drugs effective in managing type 2 diabetes mellitus, both as monotherapy and combined with other agents [3]. One member of this class, dapagliflozin, is a highly selective, potent SGLT2i, first licensed for use in Pakistan in 2017 [4,5].

Several studies have evaluated and confirmed the safety and efficacy of dapagliflozin in real-life clinical settings. Pakistani populations differ in genetic characteristics, as well as in demographic, cultural, and lifestyle characteristics, from the populations of Western countries [6-10]. This retrospective, real-life observational study evaluated the effectiveness and safety of dapagliflozin 10 and $5 \mathrm{mg}$ once daily combined with other agents in Pakistani patients.

\section{Materials And Methods}

This retrospective observational study involved patients evaluated at endocrinology clinics of Shifa 
International Hospital and Umar Diabetes and Foot Care Centre in Islamabad, Pakistan. The study was conducted after approval by Ethical Review Committee. All patients with type 2 diabetes, who were treated with dapagliflozin, were identified by screening electronic medical records at these hospitals. Patients were included if they had been diagnosed with type 2 diabetes for at least six months and were treated with dapagliflozin as monotherapy or combined with one or two other oral agents or insulin. Patients were excluded if they had type 1 diabetes, a glomerular filtration rate (GFR) $<45 \mathrm{ml} / \mathrm{min} / 1.73 \mathrm{~m}^{2}$, or a history of recurrent genitourinary tract infections. Patients were also excluded if they were taking this drug for weight loss, or if data were missing from their medical records.

Data were collected at the first visit and at follow-up. The date of the first prescription of dapagliflozin was defined as the medication index date. The baseline period was defined as two to three months before the medication index date. Based on available information, follow-up data were retrieved three to six months after the medication index date.

Patient information was collected using a predesigned data collection form. Demographic characteristics included age and gender, and disease-related characteristics at the first visit included duration of diabetes, number of comorbidities, microvascular complications (e.g., retinopathy based on retinal eye screening, nephropathy based on GFR below $90 \mathrm{ml} / \mathrm{min} / 1.73 \mathrm{~m}^{2}$, neuropathy based on symptoms), dyslipidemia, and urinary albumin:creatinine ratio. Details about other antidiabetic drugs, antihypertensive agents, and lipidlowering medications were also recorded at baseline and follow-up, as were clinical parameters, including weight, systolic blood pressure, diastolic blood pressure, glycated hemoglobin (HbA1c), and serum creatinine concentrations. For laboratory parameters, the last available measurement during follow-up (three to six months after medication index date) was compared with baseline measurements.

The safety of dapagliflozin was assessed by recording adverse effects, the proportion of patients with adverse effects, and the proportion of patients who discontinued treatment due to side effects or lack of efficacy.

\section{Statistical analysis}

All statistical analyses were performed using IBM SPSS Statistics for Windows, Version 20.0 (Armonk, NY: IBM Corp.). Categorical variables were recorded as frequencies and percentages for each category and compared by McNemar's tests, and continuous variables were recorded as means and standard deviations and compared by paired t-tests. Predictors of post-dapagliflozin HbA1c (\%) reduction and weight loss (dependent variable) were evaluated using a multivariate linear regression model. Results are expressed as odds ratios (ORs) and $95 \%$ confidence intervals (CIs). A p-value $<0.05$ was considered statistically significant.

\section{Results}

The study cohort consisted of 412 patients with type 2 diabetes (Figure 1). Table 1 summarizes their baseline characteristics. The 412 patients included 226 (54.9\%) men and 186 (45.1\%) women, with a mean age of $52.6 \pm 10.8$ years and mean diabetes duration of $9.3 \pm 6.2$ years. At baseline, their mean weight was $87.07 \pm 16.11$ $\mathrm{kg}$, and their mean $\mathrm{HbA1c}$ concentration was $9.07 \% \pm 2.07 \%$. At baseline, $88.3 \%$ of patients were being treated with a single antidiabetic agent, $11 \%$ were on dual therapy, and $33.7 \%$ were being treated with insulin.

\begin{tabular}{|c|c|}
\hline Variable & Values \\
\hline Age (years), mean $\pm S D$ & $52.6 \pm 10.8$ \\
\hline Diabetes duration (years), mean $\pm S D$ & $9.34 \pm 6.20$ \\
\hline \multicolumn{2}{|l|}{ Gender, n (\%) } \\
\hline Male & $226(54.9)$ \\
\hline Female & $186(45.1)$ \\
\hline HbA1C (\%), mean \pm SD & $9.07(2.07$ \\
\hline Weight (kg), mean $\pm S D$ & $87.07(16$ \\
\hline SBP $(\mathrm{mmHg})$, mean \pm SD & $126.38(1$ \\
\hline DBP $(\mathrm{mmHg})$, mean \pm SD & $82.34(9$ \\
\hline \multicolumn{2}{|l|}{ Comorbidity, n (\%) } \\
\hline No & $23(5.6)$ \\
\hline Yes & $289(94$ \\
\hline
\end{tabular}




\section{Cureus}

No. of comorbidities, mean \pm SD

$2.28 \pm 1.28$

Hypertension, n (\%)

$212(51.5)$

Heart diseases, n (\%)

47 (11.4)

Dyslipidemia, n (\%)

267 (64.8)

No. of concomitant medications, mean \pm SD

$6.33 \pm 2.39$

No. of antihyperglycemic medications, mean \pm SD

$3.07 \pm 0.99$

No. of antihypertensive medications, mean \pm SD

$1.08 \pm 1.01$

Dapagliflozin dose, $\mathrm{n}(\%)$

$5 \mathrm{mg}$

75 (18.2)

$10 \mathrm{mg}$

337 (81.8)

Antihypertensive drugs, $\mathrm{n}(\%)$

ACE inhibitors

Ca-channel blockers

95 (23.1)

Diuretics

$50(12.1)$

ARBs

188 (45.6)

Beta blockers

58 (14.1)

Antihyperglycemic drugs, n (\%)

None

Monotherapy

Dual therapy

Insulin

Lipid-lowering therapy, n (\%)

Statins

Simvastatin

Atorvastatin

Rosuvastatin

137 (33.3)

Pitavastatin

23 (5.6)

Other lipid-lowering medications, n (\%)

Ezetimibe

22 (5.3)

Fenofibrate

$20(4.8)$

\section{TABLE 1: Baseline characteristics of study population}

SD, standard deviation; HbA1c, glycated hemoglobin; SBP, systolic blood pressure; DBP, diastolic blood pressure; ACE, angiotensin-converting enzyme; ARB, angiotensin receptor blocker. 


\section{Cureus}

\section{Excluded:}

Patients with type 1 diabetes ( $n=67$ ),

Patients taking dapagliflozin for weight loss $(n=54)$,

Patients who have no follow-up visit data $(n=68)$,

Missing HbA1c value $(n=13)$

Patients used dapagliflozin for $<6$ months $(n=54)$

\section{Recruited}

$n=412$

\section{FIGURE 1: Study flow}

Patient flow diagram showing the profile of patients in this trial.

HbA1c, glycated hemoglobin.

Table 2 shows changes in mean clinical outcomes from the baseline (first visit) to follow-up in these patients. HbA1c concentration was significantly lower at follow-up than at baseline $(7.97 \% \pm 0.98 \mathrm{vs}$. $9.07 \% \pm 2.07$, respectively; $\mathrm{p}<0.001)$. The proportion of patients who achieved different levels of HbA1c at baseline and follow-up within six months are presented in Figure 2 . Bodyweight $(85.09 \pm 15.92 \mathrm{~kg}$ vs. $87.07 \pm 16.11 \mathrm{~kg}, \mathrm{p}<0.001)$ and diastolic blood pressure $(80.34 \pm 7.12 \mathrm{mmHg}$ vs. $82.34 \pm 9.61 \mathrm{mmHg}$, respectively; $\mathrm{p}<0.001$ ) were also significantly lower at follow-up than at baseline, whereas systolic pressure showed a marginally significant reduction $(123.5 \pm 16.57 \mathrm{mmHg}$ vs. $126.83 \pm 19.97 \mathrm{mmHg}$, respectively; $\mathrm{p}=0.048$ ).

\begin{tabular}{|c|c|c|c|c|}
\hline Variable & Baseline & Follow-up & Change & $P$ value \\
\hline Weight (kg) & $87.07 \pm 16.11$ & $85.09 \pm 15.92$ & $-1.98 \pm 3.03$ & $<0.001$ \\
\hline HbA1C (\%) & $9.07 \pm 2.07$ & $7.97 \pm 0.98$ & $-1.1 \pm 1.43$ & $<0.001$ \\
\hline SBP (mmHg) & $126.83 \pm 19.97$ & $123.5 \pm 16.57$ & $-2.80 \pm 15.67$ & 0.048 \\
\hline DBP (mmHg) & $82.34 \pm 9.61$ & $80.34 \pm 7.12$ & $-2.02 \pm 7.68$ & $<0.001$ \\
\hline
\end{tabular}

TABLE 2: Changes in clinical outcomes from baseline to three to six months after starting dapagliflozin treatment in patients with type 2 diabetes

HbA1c, glycated hemoglobin; SBP, systolic blood pressure; DBP, diastolic blood pressure. 


\section{Cureus}

- Baseline $\quad$ Follow up

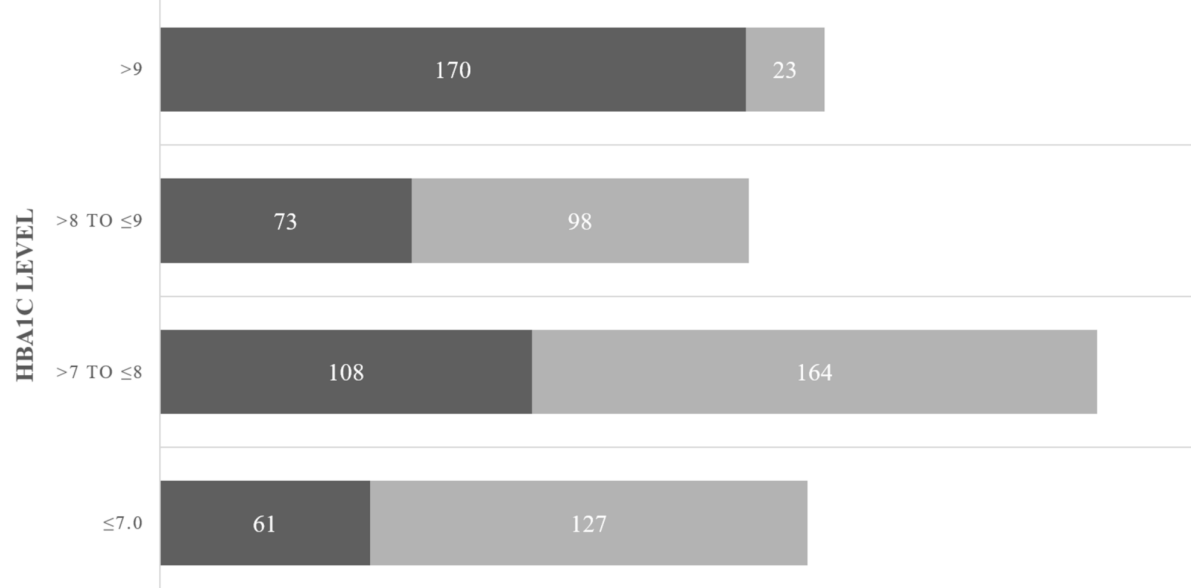

PROPORTION OF PATIENTS

\section{FIGURE 2: Levels of $\mathrm{HbA1c}$ at baseline and follow-up}

Numbers of patients achieving various $\mathrm{HbA1c}$ levels at baseline and after three to six months months of treatment with dapagliflozin.

HbA1c, glycated hemoglobin.

Of these patients, $43.7 \%$ reported that dapagliflozin had no adverse effects, whereas $27.3 \%$ reported some minor adverse effects, the most frequent being polyuria, urinary tract infections, and genital infections (Table 3). About 4\% developed hypoglycemia, whereas $29 \%$ discontinued treatment due to a lack of efficacy.

$\begin{array}{lc}\text { Variable } & \mathbf{N}(\%) \\ \text { None } & 180(43.7) \\ \text { Hypoglycemia } & 19(4.6) \\ \text { Body spasm } & 2(0.5) \\ \text { Fatigue } & 1(0.2) \\ \text { Genital infections } & 4(1.0) \\ \text { Gastrointestinal problems } & 1(0.2) \\ \text { Headaches } & 1(0.2) \\ \text { Joint pains } & 1(0.2) \\ \text { Polyurea } & 11(2.7) \\ \text { Shivering } & 1(0.2) \\ \text { Skin rashes } & 2(0.5) \\ \text { Urinary problems } & 5(1.2) \\ \text { UTI } & 5(1.2) \\ \text { Weakness } & 1(0.2) \\ \text { No benefit } & 29(7.0)\end{array}$

\section{TABLE 3: Side effects of dapagliflozin}

UTI, urinary tract infection. 


\section{Discussion}

This retrospective analysis was the first study in Pakistan to assess the safety and efficacy of an SGLT2i in patients with diabetes mellitus. Dapagliflozin initiation was found to significantly improve clinical parameters in patients with diabetes in a real-world clinical setting, reducing HbA1c concentration, weight, and blood pressure, similar to the results of clinical trials. Of the included patients, $30.8 \%$ achieved a target $\mathrm{HbA1c} \leqslant 7 \%$ after starting dapagliflozin. Moreover, the proportion of patients with $\mathrm{HbA1c}>9 \%$ decreased significantly, from $41.26 \%(\mathrm{n}=170)$ at baseline to only $5.58 \%(\mathrm{n}=23)$ at follow-up. These findings indicated that dapagliflozin-associated improvement was greater among patients with poor glycemic control, despite the study cohort being younger and having a longer duration of diabetes than other study cohorts.

One of the benefits of SGLT2 inhibitor treatment in patients with type 2 diabetes mellitus is the weight loss associated with this class of drugs, a study showed weight loss change versus placebo was -1.3 to $-2.0 \mathrm{~kg}$ [11]. In the present study, the mean weight change after dapagliflozin initiation was $-1.98 \mathrm{~kg}$, comparable to findings in a large real-world cohort, which reported a mean weight change of $-2.20 \mathrm{~kg}$ after treatment with dapagliflozin for three to six months.

Although $27 \%$ of the patients in this study experienced side effects of dapagliflozin, only a small percentage had urinary tract infections and genital infection. This may have been due to the use of wet hygiene practices in Pakistani populations, resulting in lower rates of urinary tract and genital infections than those observed in other clinical studies of dapagliflozin [12]. Also, only $4 \%$ of the participants in the present study developed hypoglycemia, similar to previous findings [13]. However, the patients in our study who developed hypoglycemia were already on insulin or a sulfonylurea, suggesting that hypoglycemia in these patients was not primarily caused by dapagliflozin.

This study had several limitations, mainly concerning the source of the data. For example, the interval between $\mathrm{HbA1c}$ measurements was not constant. Also, patient records did not mention whether insulin doses were reduced after they started taking dapagliflozin. Because this study analyzed patients treated with dapagliflozin for three to six months, it was not possible to predict therapeutic persistence. Large-scale reallife studies are needed to determine whether treated with SGLT2is or hygiene practices were responsible for the reduced rates of urinary tract and genital infections in Pakistani populations. An ongoing open-label randomized controlled trial is testing the safety and efficacy of another SGLT2i, empagliflozin, in a Pakistani Muslim population (SAFE-PAK Study).

\section{Conclusions}

This retrospective, real-life observational study evaluated the effectiveness and safety of dapagliflozin in Pakistani patients. Our findings indicate that the SGLT2i dapagliflozin is an effective agent in treating patients with type 2 diabetes, with good efficacy and safety profiles. Dapagliflozin use in patients with diabetes is associated with improved glycemic control by controlled excretion of glucose through the urine and weight loss as a secondary, additional benefit. Furthermore, it had a positive impact on the cardiovascular dynamics by maintaining blood pressure. However, dapagliflozin use is associated with a mild risk of urinary tract infection, albeit lower than what has been previously reported. Dapagliflozin is a useful treatment option for patients in this cohort with type 2 diabetes.

\section{Additional Information \\ Disclosures}

Human subjects: Consent was obtained by all participants in this study. Shifa International Hospitals Ltd issued approval 191-681-2019. Shifa International Hospitals Ltd IRB \& Ethics Committee issued approval 191-681-2019. Please note that with reference to your study entitled as "The outcome of dapaglifozin use in real life clinical settings in tertiary care hospitals of Pakistan" has been reviewed by the IRB/EC. The IRB \& Ethics Committee is pleased to approve the study. Animal subjects: All authors have confirmed that this study did not involve animal subjects or tissue. Conflicts of interest: In compliance with the ICMJE uniform disclosure form, all authors declare the following: Payment/services info: All authors have declared that no financial support was received from any organization for the submitted work. Financial relationships: All authors have declared that they have no financial relationships at present or within the previous three years with any organizations that might have an interest in the submitted work. Other relationships: All authors have declared that there are no other relationships or activities that could appear to have influenced the submitted work.

\section{References}

1. Basit A, Fawwad A, Qureshi H, Shera AS: Prevalence of diabetes, pre-diabetes and associated risk factors: second National Diabetes Survey of Pakistan (NDSP), 2016-2017. BMJ Open. 2018, 8:e020961. 10.1136/bmjopen-2017-020961

2. Beckman JA, Creager MA: Vascular complications of diabetes. Circ Res. 2016, 118:1771-1785. 10.1161/CIRCRESAHA.115.306884 
3. Davis PN, Ndefo UA, Oliver A: Dapagliflozin: a sodium glucose cotransporter 2 inhibitor for the treatment of diabetes mellitus. J Pharm Pract. 2016, 29:165-171. 10.1177/0897190014566308

4. United States Food and Drug Administration: Highlights of prescribing information for Farxiga (dapagliflozin) tablets. (2019). Accessed: December 7, 2019: https://www.accessdata.fda.gov/drugsatfda_docs/label/2019/202293s015lbl.pdf.

5. Bailey CJ, Gross JL, Hennicken D, Iqbal N, Mansfield TA, List JF: Dapagliflozin add-on to metformin in type 2 diabetes inadequately controlled with metformin: a randomized, double-blind, placebo-controlled 102week trial. BMC Med. 2013, 11:43. 10.1186/1741-7015-11-43

6. Anwar I, Hussain S, Rehman AU, Hussain M: Genetic variation among the major Pakistani populations based on 15 autosomal STR markers. Int J Legal Med. 2019, 133:1037-1038. 10.1007/s00414-018-1951-0

7. Shahzadi S, Shabana, Sarwar S, Shahid SU: Genome-wide implicated risk variants of TCF7L2 gene contribute to type 2 diabetes susceptibility by modulating serum lipids in Pakistani population. Int J Diabetes Dev Ctries. 2019, 39:302-307. 10.1007/s13410-018-0694-2

8. National Institute of Population Studies (NIPS), Pakistan and ICF. Pakistan Demographic and Health Survey 2017-18. (2019). Accessed: December 7, 2019: https://dhsprogram.com/pubs/pdf/FR354/FR354.pdf.

9. Sathar ZA, Royan R, Bongaarts J (Eds): Capturing the demographic dividend in Pakistan . UNPF/Population Council, New York; 2013.

10. Canadian Diabetes Association Clinical Practice Guidelines Expert Committee, Cheng AYY: Canadian Diabetes Association 2013 Clinical practice guidelines for the prevention and management of diabetes in Canada. Can J Diabetes. 2013, 37:S1-S3. 10.1016/j.jcjd.2013.01.009

11. List JF, Woo V, Morales E, Tang W, Fiedorek FT: Sodium-glucose cotransport inhibition with dapagliflozin in type 2 diabetes. Diabetes Care. 2009, 32:650-657. 10.2337/dc08-1863

12. Gadzhanova S, Pratt N, Roughead E: Use of SGLT2 inhibitors for diabetes and risk of infection: analysis using general practice records from the NPS MedicineWise MedicineInsight program. Diabetes Res Clin Pract. 2017, 130:180-185. 10.1016/j.diabres.2017.06.018

13. Rosenstock J, Vico M, Wei L, Salsali A, List JF: Effects of dapagliflozin, an SGLT2 inhibitor, on HbA(1c), body weight, and hypoglycemia risk in patients with type 2 diabetes inadequately controlled on pioglitazone monotherapy. Diabetes Care. 2012, 35:1473-1478. 10.2337/dc11-1693 